\title{
Refactoring Local to Cloud Data Types for Mobile Apps
}

\author{
Michael Hilton \\ Oregon State University, USA \\ hiltonm@eecs.oregonstate.edu
}

\author{
Michał Moskal \\ Microsoft Research, USA \\ Michal.Moskal@microsoft.com
}

\author{
Arpit Christi \\ Oregon State University, USA \\ christia@eecs.oregonstate.edu
}

\author{
Sebastian Burckhardt \\ Microsoft Research, USA \\ sburckha@microsoft.com
}

\author{
Danny Dig \\ Oregon State University, USA \\ digd@eecs.oregonstate.edu \\ Nikolai Tillmann
Microsoft Research, USA
nikolait@microsoft.com
}

\begin{abstract}
Mobile cloud computing can greatly enrich the capabilities of today's pervasive mobile devices. Storing data on the cloud can enable features such as automatic backup, seamless transition between multiple devices, and multiuser support for existing apps. However, the process of converting local into cloud data types requires high expertise, is difficult, and time-consuming. Refactoring techniques can greatly simplify this process.

In this paper we present a formative study where we analyzed and successfully converted four real-world touchdevelop apps into cloud-enabled apps. Based on these lessons, we designed and implemented, ClOUDIFYER, a tool that automatically refactors local data types into cloud data types on the touchdevelop platform. Our empirical evaluation on a corpus of 123 mobile apps resulting in 2722 transformations shows (i) that the refactoring is widely applicable, (ii) Cloudifyer saves human effort, and (iii) CLOUdIFYER is accurate.
\end{abstract}

Categories and Subject Descriptors D.2.7 [Software Engineering]: Distribution, Maintenance, and Enhancement

General Terms Refactoring

Keywords Refactoring, Cloud Computing, Empirical Study

\section{INTRODUCTION}

The marriage of cloud and mobile computing has transformed our access to data. If users lose their phone, upon activating a new phone, all the data (e.g., contacts, pictures) is there due to the automatic backup of data. A user can start editing a document on their mobile device and resume editing it on their tablet at home, thus seamlessly transitioning between multiple devices operating on the same data. Additionally, the cloud enables rich, multi-user apps. Examples abound from domains such as social networking (e.g., Facebook, Twitter), multiplayer games, and collaborative data collection (e.g., Citizen Science [8]).

Permission to make digital or hard copies of all or part of this work for personal or classroom use is granted without fee provided that copies are not made or distributed for profit or commercial advantage and that copies bear this notice and the full citation on the first page. To copy otherwise, to republish, to post on servers or to redistribute to lists, requires prior specific permission and/or a fee.

MOBILESoft'14, June 2-3, 2014, Hyderabad, India.

Copyright (C) $14 \mathrm{ACM} / 14 / 06 \ldots \$ 15.00$.
To take advantage of the benefits of the cloud, app developers face a high entry barrier. They need expertise on many topics: communication protocols (e.g., web services, REST, SOAP, etc.), data storage (e.g., Amazon S3, Microsoft SkyDrive, etc.), databases, cloud infrastructure (e.g., Amazon EC2, Windows Azure, etc.), programming or scripting languages. Similarly, converting a single into a multiuser app has a high entry barrier: they need to determine the candidate data structures and methods that operate on data structures as well as move them to the cloud. Currently, this process is manual, time consuming, and error prone [16].

In this paper we are lowering the entry barrier to allow even hobbyists and beginner app developers to use the cloud. Thus, we are targeting touchdevelop [32], a programming environment and language developed by Microsoft Research to write apps on mobile devices for mobile devices. We are employing automated refactoring techniques to convert local data types into cloud data types.

touchdevelop introduced specialized cloud data types [6] that provide an abstraction layer over web service implementation, communication protocols, and storage. In order to make the app responsive, even when the connection to the server is unavailable, cloud data types provide both local copies of the data as well as eventually consistent sharable cloud storage. This paradigm allows programmers to use cloud types in a similar manner to local data structures, but to also enjoy the benefits of the cloud.

In this paper we present the results of our formative study to convert local to cloud-enabled apps. We used four publicly available touchdevelop apps (three productivity tools and one game) and manually converted them into multi-user apps that share data via the cloud. In doing so, we discovered four conversion steps: (i) identify local data structures that need to be moved to the cloud, (ii) for each identified local data structure add a new cloud data structure, (iii) replace local data type API calls with cloud API calls, (iv) initialize the cloud data types. Not all these steps can be automated; some (e.g., identifying data types that need to be moved) require domain knowledge which is best provided by the app developer.

Using the lessons that we learned from the formative study, we designed and implemented a refactoring tool, ClOUDIFYER, to automate the conversion of local Number Collection into Cloud Data Table, and to transform the API calls. We selected this refactoring because its manual application is challenging for several reasons. In addition to differences in the names of the APIs, there are also differences in the cardinality of the mapping. Sometimes the mapping is 1-to-1 (e.g., count is the same for both data types), while other times the mapping is 1-to-many (e.g., insert at from Collection is transformed into a sequence of 3 operators from Table). Sometimes there is no mapping at all, in which case 
Cloudifyer injects functions to achieve the same computation. For example, collection->max needs to be converted into a function that iterates atomically over the elements of the Table.

This paper makes the following contributions:

- Idea: To the best of our knowledge, we are the first to enable hobbyists and beginner programmers to tap into the power of mobile cloud computing through the use of refactoring techniques.

- Formative Study: We have conducted a formative study on four real-world apps to learn what transformations are needed to convert single to multi-user mobile apps.

- Tool: We have designed and implemented the analysis and transformation algorithms to refactor local data types into cloud data types on the touchdevelop platform.

- Evaluation: We have evaluated our tool, Cloudifyer, on a corpus of 123 mobile apps, resulting in 2722 transformations. The results show (i) that the refactoring is widely applicable: $94 \%$ of the candidate local Collections were successfully refactored into Cloud Data Table. Second, CloudIFYER saves human effort: on average it took 3 seconds for each performed refactoring. Third, CLOUDIFYER is accurate: $100 \%$ of the applied transformations are correct, and the tool correctly identified $95 \%$ of all necessary transformations. The tool and all the data are publicly available at:

http://cope.eecs.oregonstate.edu/cloudifier

\section{BACKGROUND ON TOUCHDEVELOP AND CLOUD DATA TYPES}

touchdevelop is a programming language and integrated development environment (IDE) that allows the development of mobile applications (called scripts) on any device, for any device. It provides a development experience that is both ubiquitous and social: ubiquitous because the IDE is optimized for operation on touchscreen devices like phones or tablets, and social because scripts and libraries can be easily shared, forked, commented on, reviewed, and published in the cloud. Both touchdevelop and the touchdevelop scripts are compiled to HTML5/JavaScript, thus they can execute on all major smartphone, tablet, and personal computer brands.

The main purpose of touchdevelop is: (i) to provide an instrument for enticing and educating the next generation of programmers, and (ii) to develop novel programming language features that can simplify and accelerate the development of cloud-connected mobile applications (of which touchdevelop itself is a prime example). An example of the latter are cloud data types [6], which were recently added to touchdevelop.

Cloud types make it easy to share data between instances of the app running on multiple devices, by simply declaring such data to be of a particular cloud type. Once declared, the runtime then automatically synchronizes the data across multiple devices that are part of the same cloud session. A cloud session may include devices by a single user or by multiple users. For example, a single user may want to synchronize settings, calendars, contact lists, or any other script data between various devices she owns. Examples of multiple-user scenarios include a simple grocery list (to help family members keep track of items to be purchased on the next trip to the store), multi-player games that share game state, or applications that include typical social features like comments, reviews, achievements, high scores, and so on.

There are three categories of cloud data.

Cloud variables have a name and a type, which can be a simple type (number, string, etc.) or a reference to a cloud table row.
All cloud variables support get and set operations. Number variables also support an add operation.

Cloud tables are declared to have a name, and any number of named, typed columns. The types available for the columns are the same as for cloud variables. Tables support operations for adding a new row (always appended at the end of the table), deleting a row (deletion is permanent and idempotent), and enumerating over rows (in the order they were appended).

Cloud indexes are made up of entries that contain keys and values. All keys and values have names and types. The index contains exactly one entry for each key or combination of keys, and this entry can be retrieved using the at operation. Note that it is not possible to add or remove entries from indexes (because there is always exactly one entry per key). Entries for which all values are the default value are considered 'empty'. Indexes support enumeration of all non-empty entries. The number of non-empty entries is always finite, even if the number of entries is infinite (for example, if using a key of type string).

Cloud types are integrated into the programming language and are thus as easy to use as data types used by traditional programming languages, (simple types, objects, and collections) but their interface is more similar to types typically used for persistent storage (such as tables and indexes). The reason for this design choice is that cloud types are designed specifically to support synchronization of replicas in a distributed system with a reliable server and clients that may crash silently. In such a system, garbage collection is impossible as the server never knows if there is some client alive with a reference to a particular piece of data). The memory must thus be managed explicitly, which is facilitated by using globally named variables, tables and indexes. Also, since client devices can read and update local data at all times (whether connected or not), the server must resolve conflicts. To this end, all operations on the cloud types are designed to support automatic conflict resolution, i.e. eventual consistency can be obtained by determining a total order of conflicting updates on the server $[6,30]$. Such conflict resolution is easier when the semantics is implicit in the type instead of being encoded in arbitrary heap operations-for example, consider resolving conflicts in a table where two devices add a row, versus a linked list where two devices change a pointer's value somewhere.

As a consequence, programs that use classical heap-based object and collection data types require refactoring to take advantage of the automatic persistence and synchronization provided by cloud types. Roughly, this amounts to making classes correspond to tables, objects to rows, and fields to columns.

\subsection{Consistency Model}

As multiple clients can concurrently update the same data, even during offline operation, conflicts may need to be resolved afterthe-fact, in a manner that guarantees eventual consistency. To give the reader a general idea, we now briefly describe the mechanism used in touchdevelop, which is a simple, specialized form of eventually consistent transactions [5] and guarantees causal eventual consistency.

For each cloud session, touchdevelop stores a log of all updates performed in the cloud. The basic idea is to use what is often called primary replication: Client devices (secondary replicas) stream all locally issued updates to the touchdevelop server (primary replica). The server interleaves all updates in the order received, and stores them in log which reflects the final ordering of the updates. The server also streams the final update log back to the clients, where the updates in the log get applied to the locally stored copy of the data. However, this basic primary replication mechanism is enhanced in several ways: 
1. Local Buffers. Local updates are made visible immediately, not only after they are echoed (confirmed) by the server. Conceptually, the client maintains a buffer of unconfirmed updates, similar to store buffers in the TSO memory model, and superimposes the effect of these updates over the current confirmed state. The use of such buffers improves the perceived performance and allows seamless offline operation, but breaks strong consistency (which is a necessary consequence of allowing offline operation, as the CAP theorem $[4,14]$ shows).

2. Automatic Transactions. Updates are transmitted not individually, but as a group (called an update transaction). The transaction boundaries are determined automatically based on the event queue: any time there is no event executing (i.e. when the execution is quiesced), the current transaction is ended and a new one is started. The use of automatic transactions helps to avoid typical atomicity errors when updating multiple data items at the same time.

3. Cloud Types. The updates in the log are not just simple write operations, but mirror the rich semantics of the cloud types. Cloud types allow programmers to work around limitations of the weak consistency model, because they allow semantically meaningful conflict resolution simply by ordering the updates into a consistent order. For example, the cloud number type provides an add method in addition to the usual get and set. Applications of add from multiple clients can be meaningfully merged by the server, whereas set has the last-writer-wins semantics.

Because a weak consistency model is used, refactoring for cloud types can introduce consistency errors that were not present in the original program. The responsibility for identifying such problems and correcting them remains with the user.

Example Let's consider a client A performing two updates, $\mathrm{x}$ := $\mathrm{x}+2$ followed by $\mathrm{x}:=\mathrm{x}+4$, in an event handler E. Client $\mathrm{B}$ is executing $x:=x+3$, where $x$ is initially 0 . These are the only possible executions:

- B's update is streamed to server and then to A before E starts executing, in which case $\mathrm{x}$ will be eventually 9

- A performs its updates, setting $\mathrm{x}$ to 6 , and $\mathrm{B}$ sets $\mathrm{x}$ to 3 . Depending in which order the updates reach the server the eventual value of $x$ will be either 3 or 6 . Regardless, while $\mathrm{E}$ is executing, A will keep seeing $\mathrm{x}$ as 6 from the point of the second update on (due to local buffers above), and only in the next event it may discover that it is now in fact 3.

Note that an update from the server cannot arrive between the two updates in $\mathrm{E}$ (due to automatic transactions above). Also note that if the updates were $\mathrm{x}$->add (2); $\mathrm{x}$->add (4) for $\mathrm{A}$, and $\mathrm{x}$->add (3) for $B$, then the eventual value of $x$ would be always 9 , but A could still see $\mathrm{x}$ as 6 or 9 after its updates.

\subsection{TouchDevelop plug-ins}

The touchdevelop IDE can be extended with user-provided plugins, which are just regular touchdevelop scripts conforming to a specific interface. In general, touchdevelop scripts can query and update abstract syntax trees (ASTs) of scripts installed on the device (after asking the user for permission). A plug-in is invoked by the user when editing a script. The identifier of that script is passed to a specific action in the plug-in, that can then read and write the AST. The AST is represented as a JSON object, which can be manipulated using standard touchdevelop libraries (for purely client-side plugins), or shipped over to a cloud service of plug-in author's choice for processing.
Currently, touchdevelop supports plug-in invocation on an entire script, where the plug-in can ask the user to point to a particular AST node to operate on if necessary. In near future, we plan to have plug-ins invoked contextually for a given definition or expression.

When the user taps the plug-in invocation button, touchdevelop shows a list of currently installed scripts marked with \#scriptPlugin in their description. The user has also an option of searching touchdevelop cloud for more such scripts. We thus reuse the script distribution and rating mechanisms for plug-ins.

After plug-in execution, the user may be shown the diff with the changes performed by the plugin and asked to confirm them.

\section{FORMATIVE STUDY}

We selected four apps from the touchdevelop script bazaar for our formative study. We chose representative apps from various domains that cover different features and use cases of touchdevelop. We also looked for apps where we could envision multi-user functionality. We then converted these apps from single-user to multi-user apps. In this section we describe the script's behavior, the process to convert them, as well as the changes we needed to make.

The first app we selected was Mileage Tracker, which is publicly available (the script id can be found at [1]). Mileage Tracker is an app that records and calculates fuel usage in Miles per Gallon (MPG) and displays how it changes over time. We began by adding some functionality to the original app. We then converted Milage Tracker into a multi-user app, such that multiple family members using the same family car can collect the fuel usage even across multiple devices.

Business Manager+ [1] is an app to track business contacts. We converted it into a multi-user app so that business colleagues can share their contact list with each other.

CliffHangers [1] is a clone of the popular game "hangman." The player is presented with a series of blanks, and they must guess the letters that fill in the blanks to make a word. The player has a limited number of guesses, and if they cannot guess the word before they reach the limit, they lose the game. We converted it into a multi-player game so that two players can collaboratively work together on separate devices to guess the word.

MyAssignment [1] is an app for students to track their class assignments or projects. A single user can input information about projects, progress, due dates, etc. We converted it into a multi-user app so that multiple students can collaborate on class assignments.

After converting these apps, we ran them with multiple users on three devices to verify that in fact the apps work correctly. They are all publicly available [1].

Based on the lessons we learned from converting these four apps, we designed a process to convert a single to a multi-user app. Our process consists of four steps:

1. Identify data that needs to be moved to the cloud

2. Create new cloud data types to hold the shared data

3. Replace the local usage with cloud usage for the shared data

\section{Initialize cloud data}

Next we will illustrate these steps using the Mileage Tracker app. In step 1 we identified the data that needed to be shared: (i) the MilageRecord is a collection of numbers that holds the Miles Per Gallon for the past usage consumption, (ii) MaximumecordEntries is a number that determines how many records should be stored, (iii) UseUSUnits is a boolean that stores the preference between metric or imperial units.

To illustrate step 2, let us consider one of the shared data types, the MilageRecord collection. We created a new Cloud Data Table named MileageRecordTable to hold the data. Notice that the 
original data is stored in a one-dimensional data structure, whereas the Cloud Data Table is a two-dimensional data structure.

In step 3 we replaced all the uses of the local MilageRecord with uses of MileageRecordTable.

In step 4 we initialized MileageRecordTable. Since the data is now persistent on the cloud, we need to change the initialization code from eager to lazy in order to avoid erasing all the data every time the app is launched. In addition, we need to make a choice between using the cloud (i) as a backup for the data for one user across multiple devices("just me session") or (ii) to enable collaboration between multiple users across multiple devices ("everyone session"). These changes required adding 3 LOC and updating 2 LOC.

Notice that steps 1 and 4 require domain knowledge in order to choose and refine the proper end-user experience.

Based on the type of the input as well as the local data that we needed to migrate to cloud, we have identified three kinds of refactorings. Each refactoring can be performed using the general steps that we identified above. First, we refactored primitive data types into the corresponding cloud-enabled primitive type (e.g., from Number to Cloud Number). Second, we refactored local Data Table into Cloud Data Table. Third, we refactored local Collection into Cloud Data Table.

When carrying out the refactorings, we noticed that steps 1 and 4 are hard to automate as they require understanding the original program and the desired end-user experience. It took the second author an average of 6 minutes to perform steps 1 and 4 for each app in the formative study. Steps 2 and 3 have different degrees of complexity. The first two kinds of refactorings are trivial because there is a perfect match between the local data and the cloud data type, so the change is as simple as prepending the keyword Cloud to the variable declaration.

The third kind of refactoring is non-trivial: it requires changing the program from using a flat, one-dimensional Collection to a twodimensional Cloud Data Table, as shown in Figure 1. The APIs are different enough that sometimes we needed to map one function call from Collection into a sequence of calls from Table, whereas other times we had to augment the API by writing new functions.

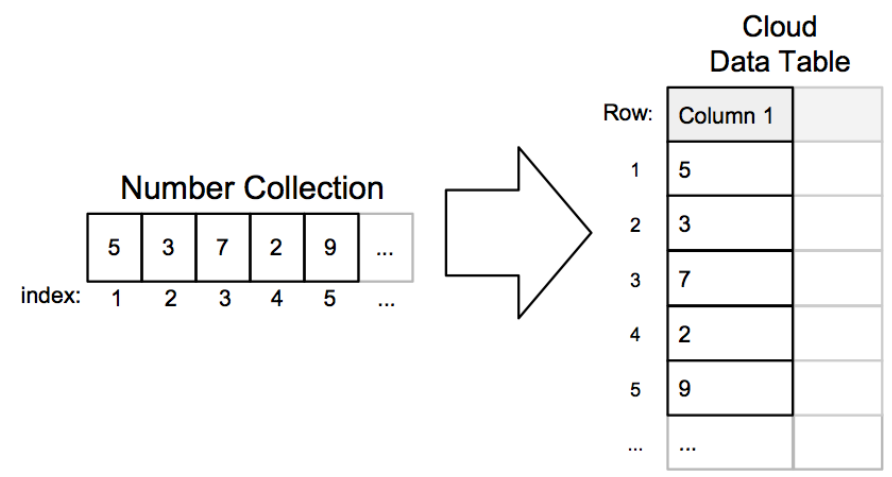

Figure 1. Conversion from Number Collection to Cloud Data Table

For these reasons, we automated this refactoring.

Table 1 lists the total number of refactorings that we applied as part of our formative study.

\section{AUTOMATED REFACTORING}

This section presents our automated refactoring which converts from a local Number Collection to a Cloud Data Table. This refactoring is composed of several transformations. The transformations are the individual changes that CLOUDIFYER must apply to the pro-

Table 1 Refactorings used in the formative study
\begin{tabular}{|c|c|c|c|}
\hline $\begin{array}{c}\text { App } \\
\text { Name }\end{array}$ & $\begin{array}{c}\text { Primitive } \\
\text { to Cloud }\end{array}$ & $\begin{array}{c}\text { Local Table to } \\
\text { Cloud Table }\end{array}$ & $\begin{array}{c}\text { Collection } \\
\text { to Cloud }\end{array}$ \\
\hline MileageTracker & 2 & 0 & 1 \\
\hline Buisness Manager+ & 0 & 0 & 5 \\
\hline CliffHangers & 8 & 0 & 2 \\
\hline My Assignments & 0 & 1 & 0 \\
\hline \hline Total & 10 & 1 & 8 \\
\hline
\end{tabular}

gram in order to accomplish this refactoring. We implemented the refactoring in a tool, CLOUdIFYER, which itself is a touchdevelop app.

Section 4.1 presents the workflow of using the tool. Section 4.2 presents the preconditions that must be true before applying the refactoring. Section 4.3 presents the three kinds of transformations needed to perform the refactoring.

\subsection{Workflow}

CLOUDIFYER is implemented as a touchdevelop app. It can be run using the touchdevelop platform like any other touchdevelop app. Once it is run, the app first prompts the user to select a script on which to perform the refactoring. Once the user has selected a script, CLOUdIFYER will display all the possible refactoring targets for the chosen script, and allow the user to choose which Number Collection they wish to convert to a Cloud Data Table. CLOUDIFYER will then perform all the needed transformations on the selected Number Collection in an automated fashion.

\subsection{Preconditions}

In the current implementation of touchdevelop, there are some differences in how Number Collections and Cloud Data Tables are treated. For example, an instance of a Number Collection can be passed as an argument or returned from a touchdevelop function. However, Cloud Data Tables cannot be passed as function arguments, or returned from functions. Also, Number Collections can be assigned to a variable, where as Cloud Data Tables cannot. Based on these differences, we developed two preconditions that must be met before CLOUDIFYER can perform a refactoring. These preconditions are inherent to how the touchdevelop language works, not limitations of our tool.

(P1) A Number Collection instance must not be passed as an argument or returned from a function.

(P2) A Number Collection instance must not be assigned to a variable.

\subsection{Transformations}

There are four categories of transformations to refactor Number Collection to Cloud Data Tables: (i) creating Cloud Data Table data structure, (ii) direct mapping of APIs, (iii) indirect mapping of APIs, and (iv) injected function to augment missing APIs. Table 2 displays the mapping between the APIs of the two data types.

We will illustrate the transformations using simplified code snippets from the MileageTracker app that we introduced in the Formative Study (see Section 3), an app that enables users to track their fuel usage. Figure 2 shows the code before and after the refactoring. The left column shows the original code. Line 1 shows the declaration of the MileageRecord Number Collection, which is the target of the refactoring. We show four functions to display all mileage record, clear existing mileage record, add a new entry to the mileage collection, and compute and display the average mileage. 


Table 2 Number Collection API's and corresponding Cloud Data Table Operations.
\begin{tabular}{|c|c|c|}
\hline Number Collection Operations & Cloud Table Operations & Transformation Type \\
\hline clear & clear & Direct \\
\hline count & count & Direct \\
\hline post to wall & post to wall & Direct \\
\hline add & add row & Indirect \\
\hline at & row at & Indirect \\
\hline set at & row at $\rightarrow$ valueName & Indirect \\
\hline remove at & row at $\rightarrow$ deleteRow & Indirect \\
\hline insert at & row at $\rightarrow$ value & Indirect \\
\hline add many & NONE & Injected Function \\
\hline avg & NONE & Injected Function \\
\hline contains & NONE & Injected Function \\
\hline index of & NONE & Injected Function \\
\hline max & NONE & Injected Function \\
\hline min & NONE & Injected Function \\
\hline random & NONE & Injected Function \\
\hline remove & NONE & Injected Function \\
\hline reverse & NONE & Injected Function \\
\hline sort & NONE & Injected Function \\
\hline sum & NONE & Injected Function \\
\hline
\end{tabular}

Create Cloud Data Table data structure: Lines 1-3 of the right-hand side of Figure 2 show the newly created data structure, MileageRecordTable. We append the Table to the end of the name for clarity. This Table contains one column, MileageRecordColumn. We will use this column to store the values that were originally stored in the MileageRecord collection.

Direct Transformations: The simplest transformations are those when the mapping from the Number Collection to Cloud Data Table APIs is one-to-one and both data types use the same name (e.g., post to wall). In this case, the transformation consists of simply replacing the receiver object. Figure 2 (a) line 8 shows the API call before the transformation, while Fig. 2 (b) line 8 shows the code after the transformation.

Indirect Transformations: Indirect Transformations are more complex than Direct Transformations, and cannot be performed with a simple find-and-replace technique. We use Indirect Transformations when the same functionality can be accomplished in both data types, but the APIs are different: the cardinality and/or the name is different. Sometimes, the transformation maps one API call from the Number Collection into a sequence of API calls from the Cloud Data Table. In addition, sometimes it turns a function call into an assignment, further increasing complexity.

One example of an Indirect Transformation is shown in Figure 2. The function AddMileageToRecord adds a number to the MileageRecord Number Collection. Line 26 in column (a) shows Mileage being added via the API call add. In order to correctly perform the transformation, Mileage now needs to be added to the Cloud Data Table MileageRecordTable. This becomes a two step process. Step 1 is to add a new row to the Cloud Data Table, and step two is to assign the value of Mileage to that row. This is shown in column (b) lines 26-27.

Injected Function Transformations: We used Injected Function Transformations when some API functionality in Number Collection cannot be replicated using APIs from Cloud Data Table. In order to transform such missing APIs, CLOUDIFYER inserts Injected Functions that provide the same functionality. Since touchdevelop does not allow Cloud Data Tables to be passed to functions, but instead must be used as a global variable, CLOUDIFYER cannot create generic functions to replicate the API functionality. Instead, it must inject a function for each Cloud Data Table that has been created as a result of a refactoring. We developed a template for each Injected Function Transformation. These templates have "holes" and ClOUdIFYER then fills up these "holes" with the relevant table name and column name. Thus CLOUDIFYER generates these Injected Functions fully automatically.

One example of an Injected Function Transformation is shown in Fig 2 (a) on lines 32-33. The function DisplayAvgMileage calculates the average value of all contents of MileageRecord, and then displays it. Once CLOUDIFYER refactors the program to use a Cloud Data Table to store the data, there is no longer an API call that will calculate the average value of all contents of a Cloud Data Table. Instead, CLOUDIFYER must introduce the injected function MileageRecordTable avg (see Fig. 2 (b) lines 36-45) that will compute the average of all the values in the MileageRecordTable. Now the API call in DisplayAvgMileage is transformed to call this avg injected function instead, as seen in Fig. 2 (b), line 32.

Another example of an Injected Function Transformation is shown in Fig 3. Column (a) shows the template for the function that calculates the maximum value of a Cloud Data Table. Column (b) shows the same function once it has been injected in the Mileage Tracker app.

Concurrency: Since multiple users can access the cloud data at the same time and perform updates concurrently, one needs to worry about the atomicity of compound operations in our Injected Functions. However, touchdevelop guarantees atomicity at the function level, thus our Injected Functions will execute atomically.

\subsubsection{Tool Limitations}

The current version of CLOUDIFYER does not support the injected function transformation needed to transform the API call add many. We believe that this function could be implemented but it remains as future work.

So far, we implemented CLOUDIFYER only for Number Collections. In order to add other types of collections, we would need to write new injected functions in order to provide additional functionality. For example, in order to support the String Collection, we 


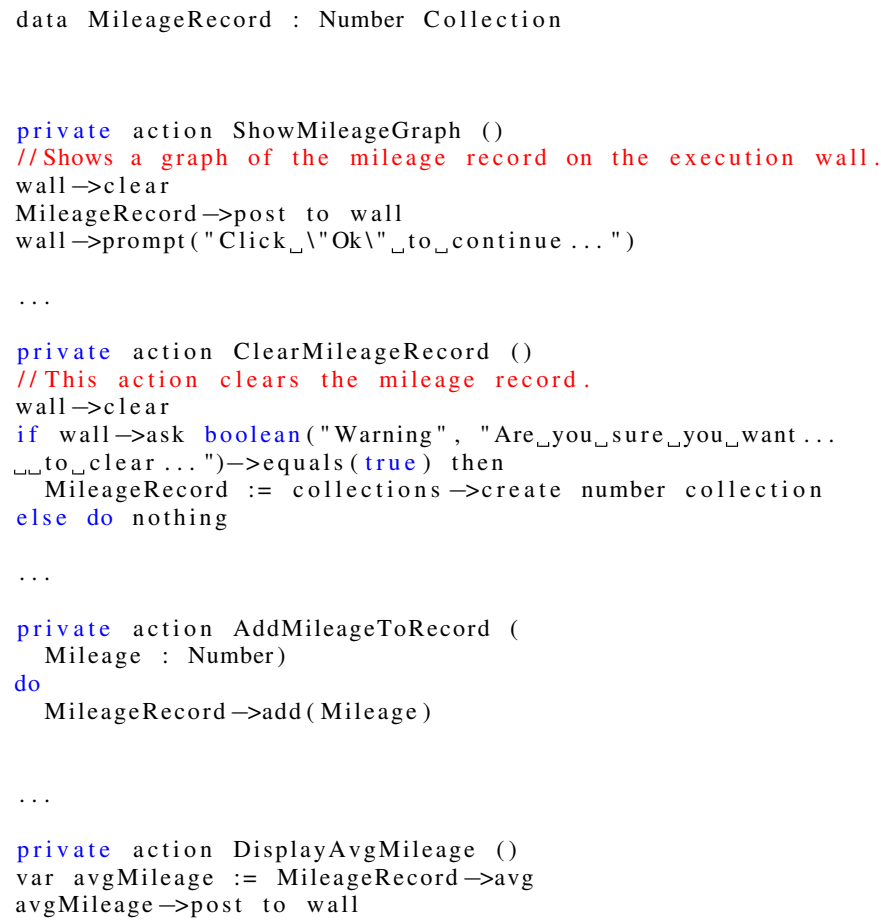

(a) before

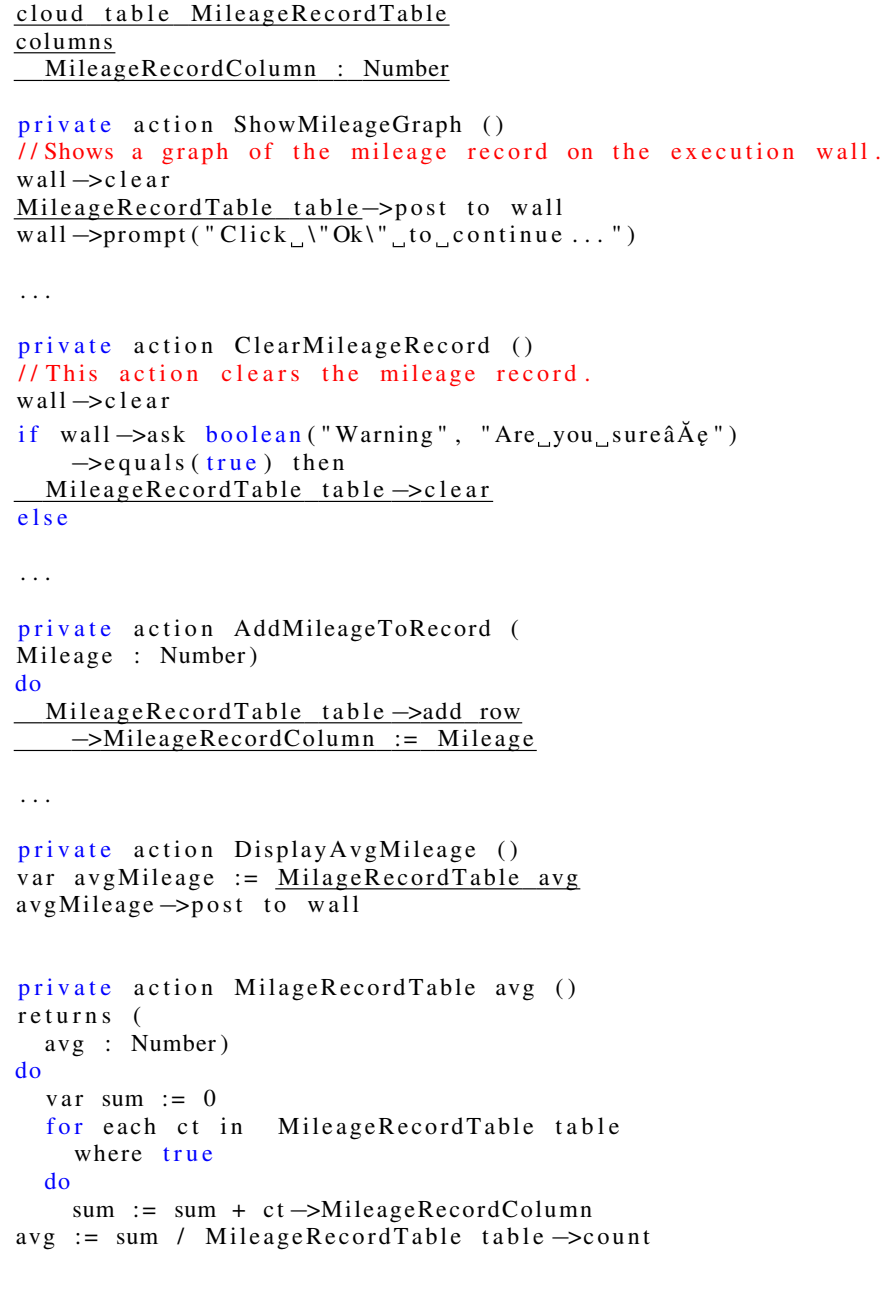

(b) after

Figure 2. Relevant code from Mileage Tracker app. The left-hand side shows the original app, whereas the right-hand side shows the refactored code. The modified code is underlined, and the avg is a newly injected function.

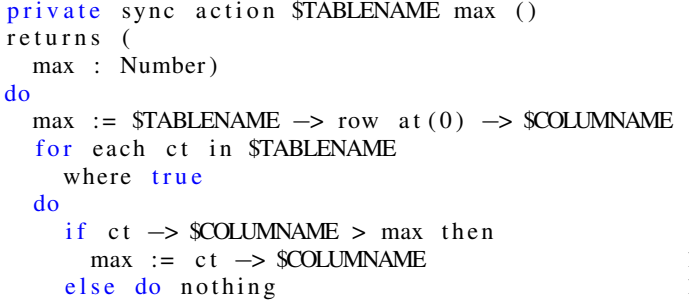

(a) Template Function

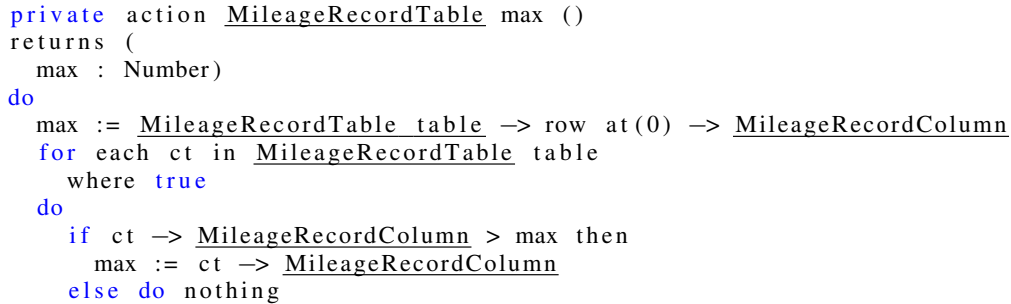

(b) Injected Function

Figure 3. Template showing the injected function max. The left hand-side shows the template with "holes", whereas the right-hand side shows the function instantiated to use the MileageRecordTable. 
would need to write an equivalent injected function for the join API method which concatenates the entire collection into one string.

\subsubsection{Implementation}

CLOUdIFYER refactors touchdevelop scripts in place. First, CloudIFYER retrieves the source of the target app as an Abstract Syntax Tree (AST) stored in JSON format from the touchdevelop script bazaar. It then transforms the AST as needed. Once all the transformations are performed, CLOUDIFYER completes the refactoring by saving the new AST for the target app.

\section{EVALUATION}

To determine if CLOUDIFYER is useful we ask the following research questions.

Q1: APPLICABILITY: How applicable is the refactoring?

Q2: EFFORT: How much effort is saved by CLOUDIFYER when refactoring?

Q3: ACCURACY: How accurate is CLOUDIFYER when performing a refactoring?

\subsection{Methodology}

To create a corpus, we wrote queries against a database of all publicly available scripts in the touchdevelop script bazaar. We wanted to collect popular and mature scripts. Thus, our query returned top scripts (based on the number of times that each script was executed) that contained a Number Collection. We retained the top 123 scripts.

Table 3 provides details about our corpus. We report the size in non-blank source lines of code (SLOC) and complexity in number of functions. We also report the number of times the apps were run by the touchdevelop community, as well as the number of installations. Notice that a user can run an app without installing it in the user profile; this feature allows a touchdevelop user to try out an app before installing it.

\begin{tabular}{lclcl}
\hline \multicolumn{5}{l}{ Table 3 Corpus Characteristics } \\
\hline \multicolumn{5}{l}{} \\
\hline Average & Max & Std Dev & Total \\
\hline SLOC & 403 & 2,526 & 483 & 47,185 \\
Functions & 21 & 121 & 26 & 2,502 \\
App Runs & 46 & 750 & 127 & 5,350 \\
App Installations & 5 & 125 & 18 & 635 \\
& & & & \\
\hline
\end{tabular}

Table 4 shows the total usage of the Number Collection APIs in our corpus. For each API method we report (i) the total number of call sites in our corpus, and (ii) the percentage of call sites among the number of Number Collection API calls.

We ran the refactoring in batch mode. Steps 1 and 4 of our process require domain knowledge. For Step 1, we refactored all the Number Collections in our corpus instead of trying to identify which data makes sense to be moved to the cloud. For Step 4, we used the same policy that the app currently uses for data initialization, as changing that would involve making design decisions for each app. As far as the data session, we used the default session provided by touchdevelop, the "just me session."

In order to determine the applicability of CLOUDIFYER, we report the number of scripts that our tool was able to refactor successfully, as well as the number of each kind of transformations performed inside a refactoring.

In order to determine the effort saved by using this refactoring tool we record the number of seconds each refactoring took.
Table 4 Usage of Number Collection API reported in number of call sites

\begin{tabular}{lcl}
\hline API Name & \# Call Sites & $\begin{array}{l}\text { \% of total Number Collection } \\
\text { API Call Sites }\end{array}$ \\
\hline At & 1010 & 39.25 \\
Set At & 632 & 24.56 \\
Add & 532 & 20.68 \\
Count & 109 & 4.24 \\
Contains & 107 & 4.16 \\
Clear & 84 & 3.26 \\
Remove At & 53 & 2.06 \\
Sum & 12 & 0.47 \\
Avg & 10 & 0.39 \\
Sort & 8 & 0.31 \\
Min & 4 & 0.16 \\
Post To Wall & 4 & 0.16 \\
Reverse & 4 & 0.16 \\
Max & 3 & 0.12 \\
Random & 1 & 0.04 \\
& &
\end{tabular}

In order to validate the accuracy of CLOUDIFYER, we randomly chose 20 scripts from the corpus of 123 refactored scripts. We ran the scripts and exercised the features for three minutes before and after each refactoring to ensure that the refactorings did not change the scripts' runtime behavior. Indeed, we did not observe any runtime differences before and after the refactoring.

In addition to running these 20 scripts we also carefully inspected the refactored code. We counted transformations that CLOUDIFYER (i) applied correctly, (ii) applied incorrectly, and (iii) missed. The first two authors manually refactored these same 20 scripts, and created a set of transformations for these refactorings. We define this set of transformations that an expert would apply as our gold standard. For each transformation that CLOUDIFYER applied, we manually compared the result of the automated transformation with the gold standard to determine if that transformation had been applied correctly. We define a TruePositive as a transformation that Cloudifyer identified and applied correctly. We define FalsePositive as a transformation that CLOUDIFYER applies to the code, but it is not part of the gold standard. In other words, CLOUDIFYER transformed code that should not have been transformed. We define a FalseNegative as a transformation that CLOUDIFYER did not apply, but it is in the gold standard. In other words, CLOUDIFYER missed applying a needed transformation.

Using these metrics we calculate precision and recall for CLOUDIFYER, using the standard definitions:

$$
\begin{gathered}
\text { precision }=\frac{\mid \text { TruePositive } \mid}{\mid \text { TruePositive }|+| \text { FalsePositive } \mid} \\
\text { recall }=\frac{\mid \text { TruePositive } \mid}{\mid \text { TruePositive }|+| \text { FalseNegative } \mid}
\end{gathered}
$$

\subsection{Results}

Next we present the results for each of the three research questions.

Applicability: Table 5 shows the results for running CLOUDIFYER on 123 scripts. Out of the 123 scripts that CLOUDIFYER attempted to refactor, $116(94 \%)$ met the preconditions for the refactoring.

Only 7 scripts were unable to be refactored. Out of these, 4 scripts did not meet precondition (P1) (the Number Collection was passed as an argument to a function), and 1 did not meet precondition (P2) (Number Collection was assigned to a local variable). The remaining 2 scripts exposed a limitation of the 
current implementation of CLOUDIFYER (they were using a currently unsupported function, add many). Thus, we think that our refactoring is widely applicable to touchdevelop scripts that contain the Number Collection data type.

\section{Table 5 Total Scripts Refactored}

\begin{tabular}{ll}
\hline Total Scripts & 123 \\
Number Successfully Refactored & 116 \\
Failed Precondition (P1) & 4 \\
Failed Precondition (P2) & 1 \\
Tool Limitations & 2
\end{tabular}

Effort: Table 6 shows the number of transformations performed per each refactoring. The first three rows show the type of these transformations (i.e., direct, indirect, and injected function). The fourth row shows the total for all types of transformations, while the last row shows the running time for each refactoring.

The first column shows the average per script, while the last column shows the total for all the scripts. Each script had, on average, 24 transformations applied in 3 seconds. Given that the touchdevelop editing experience is optimized for small screens on touch devices, our refactoring tool saves significant effort.

Moreover, most of these transformations are non-trivial. Figure 4 shows the percentage of each kind of transformation applied. By far the most common transformation kind is Indirect Transformation, which involve mapping one API method call to a sequence of one or more API method calls that do not share a common name with the original method. Thus, we believe that automation saves significant effort.

\begin{tabular}{lclcl}
\hline \multicolumn{6}{l}{ Table 6 Refactorings Applied Per Script } & & \\
\hline & Avg & Max & Std Dev & Total \\
\hline Refactorings & 1.84 & 8 & 1.38 & 211 \\
\hline Direct Trans & 2.22 & 30 & 4.45 & 249 \\
Indirect Trans & 19.88 & 466 & 56.62 & 2227 \\
Injected Function & 2.20 & 30 & 5.01 & 246 \\
\hline Total Trans & 24.30 & 466 & 56.56 & 2722 \\
\hline Time [sec] & 2.64 & 27 & 5.18 & 296
\end{tabular}

\section{Accuracy:}

By manually checking refactorings applied in 20 scripts, we found CLOUDIFYER's precision to be $100 \%$ and its recall $94 \%$. This means that all the transformations that CLOUDIFYER applied are correct. However, CLOUDIFYER missed 8 potential transformations out 145 transformations that it should have applied. We identified the root cause in our current implementation, and we expect a future version will fix this.

\subsection{Threats to validity}

Construct Validity: Do our metrics indeed measure the advantages of using ClOUdIFYER? Can we measure development effort by simply counting the time and number of transformations per refactoring? Ideally, we would have performed an experiment with real touchdevelop developers and observe them while refactoring. However, given the cutting edge nature of the Cloud Data Types, we could not find such developers. Thus, we chose to use indirect metrics, as it is commonly done in the literature [15, 33]. Second, are we sure that the performed refactorings are accurate? Ideally, we should have run tests before and after each refactoring. However, very few scripts in touchdevelop have any tests at all. In order to mitigate this we manually inspected a random set of 20 apps and

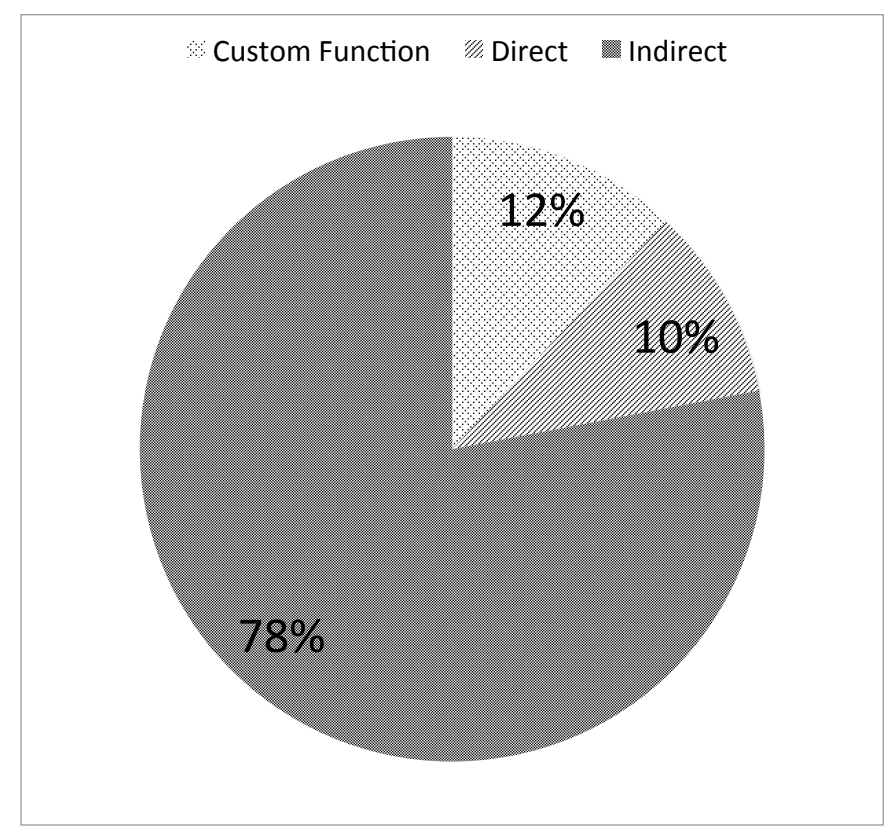

Figure 4. Transformations by Percentage

exercised their functionality to determine that the runtime behavior did not change.

Internal Validity: How did we mitigate bias during manual inspection? We randomly sampled from the set of our corpus, until we had sampled from a variety of app domains. Also, we carefully constructed the set of transformations in the gold standard before we applied CLOUDIFYER. The authors are all experts on the touchdevelop platform.

Also, the corpus that we used in the evaluation predates the introduction of Cloud Data Types, and thus the developers were unaware of our later study and could not bias their code to help CLOUDIFYER.

External Validity: Do our results generalize? For our corpus, we used 123 of the most commonly run scripts, but we put no restrictions on the type of the scripts. Our corpus includes scripts from various domains: entertainment, sports, games, education, productivity, etc. Moreover, the apps in our corpus are developed by 83 authors from a diverse end-user community. We do not foresee any reasons why our results would not generalize to other touchdevelop apps. Currently there is no widely accepted standard for cloud data types. In order for our results to generalize, cloud storage providers will first need to agree on Cloud Data Storage standards.

Reliability: Is our evaluation reliable? The apps that we used to evaluate our tool are all available on the touchdevelop bazaar, and CLOUdIFYer is published on the touchdevelop website. All these URLs are available on our webpage:

http://cope.eecs.oregonstate.edu/cloudifier

\section{DISCUSSION}

There are two reasons why touchdevelop is well suited for refactoring to the cloud. The first is that cloud types in touchdevelop have well-defined but constrained semantics which includes the eventual consistency model as described in section 2.1. The second is that touchdevelop does not allow Number Collection data objects to be aliased. 
In order to generalize our results beyond touchdevelop, one must deal with the problem of aliasing. This is a problem when the object that is being moved is referenced by another alias in the code. Consider a local object that one wishes to convert into a cloud data type. If there are aliases to the local reference, any subsequent method calls via that alias will cause the application to crash, unless one finds all aliases and changes their data type as well. The reason this is not a concern for our tool is that touchdevelop only allows cloud data types to be global objects, therefore ensuring there are no aliases to the object that was moved to the cloud.

In order to deal with these issues in environments that allow aliases (e.g., in Java), researchers [31] have been forced to implement distributed shared memory via a combination of code transformation/generation.

\section{RELATED WORK}

We group the related work in the following categories: (i) cloudrelated refactorings, (ii) multi-user apps, and (iii) tools for the end-user development community.

Refactoring and the Cloud: There is a lot of research on the generic topic of refactoring. While the original research was using refactoring to improve the design of existing programs, the more recent work used refactoring techniques to retrofit concurrency [11, 34], functional features [15], etc. As far as we know, we present the first automated tool to introduce mobile cloud computing via an automated refactoring tool.

The closest research to our current work is by Kwon and Tilevich [19] who proposed Cloud Refactoring, a tool to refactor methods of an enterprise application system into services that can be ported to the cloud. Their tool also determines if a method is a good candidate for refactoring using static and dynamic analysis. However, even though their tool automatically refactors methods into services, the programmer must still move them to the cloud manually. Our tool, CLOUDIFYER, performs the refactoring fully automatically, including moving data to the cloud.

Strauch et al. [28] describe a methodology for refactoring applications to move local data to cloud data. They propose several cloud data patterns, and describe migration scenarios for these patterns. Although this work provides a methodology at a high level, the application of the refactoring is completely manual and left up to the programmer. Ling et al. [22] describe a systematic refactoring approach using category theory to formally define an approach to convert Object Oriented systems into Service-Oriented Architecture systems. Our work is different from these in that we provide a tool to perform the refactoring in an automated manner.

Researchers have used many approaches to harness the power of the cloud. One of the more common uses of the mobile cloud is to offload computing in order to make apps more energy efficient and thereby preserve battery life, such as Spectra [12], Slingshot [29], and MAUI [10]. Kwon and Tilevich [18] proposed an approach to offload computation to the cloud to save battery life that is tolerant to network outages or unavailability.

CloudCone [7] also attempts to harness the power of mobile cloud computing, but their approach is significantly different then ours in that they create clones on the cloud, and then move the execution of the application as well as it's state between the cloud and the device.

Researchers $[3,26]$ have also used refactoring techniques to migrate from a version of the library to another version. Balaban et al. [3] propose refactorings that automatically replace obsolete library classes with their newer counterparts. Component developers have to provide mappings between legacy classes and their replacements, and an analysis based on type constraints determines where the replacement can be done. Nguyen et al. [26] designed a technique to identify API changes automatically. Their analysis compares two library versions and extracts knowledge about how the updated client code has migrated to the newer version of the API. Since this line of work is investigating API migration between two versions of the same class, the changes are less dramatic than in the case of migrating between a one-dimensional data type (Number Collection) and a two-dimensional data type (Cloud Data Table). Such a dramatic API change requires custom program transformations, and cannot be specified with a generic rule system.

Multi-user apps: Many have studied multi-user apps in the following domains: collaboration [23, 36], gaming [20], Geospatial Applications [13] (e.g., Google earth), information sharing [25], and music [27]. This research focuses on how multi-user technologies impact human interactions. In contrast, we are focused on the technical challenges of refactoring from single to multi-user apps.

End-user Development community: Lewis et al. [21] describe the need of End User Programming tools to make End User Programming more acceptable.

touchdevelop is a programming platform developed by Microsoft Research that allows hobbyist, novice programmers and end users to program on their phone for their phone. There are other programming environments that are targeted to a similar audience, including: App Inventor [35], Scratch [24], Alice [9], and Greenfoot [17]. Our recent work [2] provides refactoring tools for users of Excel. However, our current paper is the first paper to engage the mobile end-user in a refactoring workflow.

\section{CONCLUSION}

Mobile cloud computing can be harnessed to enable rich access to data. In this paper we presented a formative study to convert four apps to use the touchdevelop cloud data types. Based on these lessons, we designed, implemented, and evaluated a tool, CLOUdIFYER, to refactor local data types into cloud data types. Our empirical evaluation on 123 real apps, shows that the tool is applicable, relevant, and saves human effort. Using our refactoring tool in combination with a well designed, powerful end-user programming platform (touchdevelop) can enable even novice programmers to take advantage of the power of the cloud.

\section{ACKNOWLEDGEMENTS}

We would like to thank Caius Brindescu, Mihai Codoban, Sergii Shmarkatiuk, Alexandru Gyori, Semih Okur, Yu Lin, Cosmin Radoi, Eli Tilevich, and the anonymous reviewers for feedback on earlier drafts of this paper. This research is partly funded through NSF CCF1213091 and CCF-1219027 grants, a SEIF award from Microsoft, and a gift grant from Intel.

\section{REFERENCES}

[1] Refactoring to cloud data. March '14, http://cope.eecs. oregonstate.edu/cloudifier/.

[2] S. Badame and D. Dig. Refactoring meets spreadsheet formulas. In 28th IEEE International Conference on Software Maintenance (ICSM), pages 399-409, 2012.

[3] I. Balaban, F. Tip, and R. M. Fuhrer. Refactoring support for class library migration. In ACM International Conference on Object Oriented Programming Systems Languages and Applications (OOPSLA), pages 265-279, 2005.

[4] E. A. Brewer. Towards robust distributed systems (abstract). In 19th ACM SIGACT-SIGOPS Symposium on Principles of Distributed Computing (PODC), pages 7-, 2000.

[5] S. Burckhardt, M. Fähndrich, D. Leijen, and M. Sagiv. Eventually Consistent Transactions. In European Symposium on Programming 
(ESOP), (extended version available as Microsoft Tech Report MSRTR-2011-117), LNCS, volume 7211, pages 64-83, 2012.

[6] S. Burckhardt, M. Fähndrich, D. Leijen, and B. P. Wood. Cloud types for eventual consistency. In European Conference on Object-Oriented Programming (ECOOP), pages 283-307. 2012.

[7] B.-G. Chun, S. Ihm, P. Maniatis, M. Naik, and A. Patti. Clonecloud: elastic execution between mobile device and cloud. In 6th conference on Computer systems (EuroSys), pages 301-314. ACM, 2011.

[8] J. P. Cohn. Citizen science: Can volunteers do real research? BioScience, 58(3):192-197, 2008.

[9] S. Cooper. The design of alice. ACM Transactions on Computing Education (TOCE), 10(4):15, 2010.

[10] E. Cuervo, A. Balasubramanian, D.-k. Cho, A. Wolman, S. Saroiu, R. Chandra, and P. Bahl. Maui: making smartphones last longer with code offload. In 8th international conference on Mobile systems, applications, and services (MobiSys), pages 49-62. ACM, 2010.

[11] D. Dig, J. Marrero, and M. D. Ernst. Refactoring sequential java code for concurrency via concurrent libraries. In 31st International Conference on Software Engineering (ICSE), pages 397-407. IEEE Computer Society, 2009.

[12] J. Flinn, S. Park, and M. Satyanarayanan. Balancing performance, energy, and quality in pervasive computing. In 22nd International Conference on Distributed Computing Systems (ICDCS), pages 217 226. IEEE, 2002.

[13] C. Forlines, A. Esenther, C. Shen, D. Wigdor, and K. Ryall. Multi-user, multi-display interaction with a single-user, single-display geospatial application. In 19th Annual ACM Symposium on User Interface Software and Technology (UIST), pages 273-276. ACM, 2006.

[14] S. Gilbert and N. Lynch. Brewer's conjecture and the feasibility of consistent, available, partition-tolerant web services. SIGACT News, 33:51-59, June 2002.

[15] A. Gyori, L. Franklin, D. Dig, and J. Lahoda. Crossing the gap from imperative to functional programming through refactoring. In 9 th joint meeting of the European software engineering conference and the ACM SIGSOFT symposium on The foundations of software engineering (ESEC/FSE), pages 543-553, 2013.

[16] A. Khan, M. Othman, S. Madani, and S. Khan. A survey of mobile cloud computing application models.

[17] M. Kölling. The greenfoot programming environment. ACM Transactions on Computing Education (TOCE), 10(4):14, 2010.

[18] Y.-W. Kwon and E. Tilevich. Energy-efficient and fault-tolerant distributed mobile execution. In IEEE 32nd International Conference on Distributed Computing Systems (ICDCS), pages 586-595. IEEE, 2012.

[19] Y.-W. Kwon and E. Tilevich. Cloud refactoring: automated transitioning to cloud-based services. Automated Software Engineering, pages 1-28, 2013.

[20] K. Leichtenstern and E. André. Studying multi-user settings for pervasive games. In 11th International Conference on Human-Computer Interaction with Mobile Devices and Services (MobileHCI), pages 25:1-25:10. ACM, 2009.

[21] G. Lewis, D. Smith, L. Bass, and B. Myers. Report of the workshop on software engineering foundations for end-user programming. ACM SIGSOFT Software Engineering Notes, 34(5):51-54, 2009.

[22] H. Ling, X. Zhou, and Y. Zheng. Refactoring from object-oriented systems to service-oriented systems: A categorical approach. In
International Conference on Service Sciences (ICSS), pages 214-218. IEEE, 2010.

[23] R. Lopez-Gulliver, H. Tochigi, T. Sato, M. Suzuki, and N. Hagita. Senseweb: Collaborative image classification in a multi-user interaction environment. In 12th Annual ACM International Conference on Multimedia (MM), pages 456-459. ACM, 2004.

[24] J. Maloney, M. Resnick, N. Rusk, B. Silverman, and E. Eastmond. The scratch programming language and environment. ACM Transactions on Computing Education (TOCE), 10(4):16, 2010.

[25] M. A. Nacenta, M. R. Jakobsen, R. Dautriche, U. Hinrichs, M. Dörk, J. Haber, and S. Carpendale. The lunchtable: A multi-user, multidisplay system for information sharing in casual group interactions. In 2012 International Symposium on Pervasive Displays (PerDis), pages 18:1-18:6. ACM, 2012.

[26] H. A. Nguyen, T. T. Nguyen, G. Wilson, Jr., A. T. Nguyen, M. Kim, and T. N. Nguyen. A graph-based approach to api usage adaptation. In $A C M$ International Conference on Object Oriented Programming Systems Languages and Applications (OOPSLA), pages 302-321. ACM, 2010.

[27] H. Sørensen and J. Kjeldskov. The interaction space of a multi-device, multi-user music experience. In 7th Nordic Conference on HumanComputer Interaction: Making Sense Through Design (NordiCHI), pages 504-513. ACM, 2012.

[28] S. Strauch, V. Andrikopoulos, and T. Bachmann. Migrating application data to the cloud using cloud data. In e 3rd International Conference on Cloud Computing and Service Science, (CLOSER), pages 36-46. SciTePress, 2013.

[29] Y.-Y. Su and J. Flinn. Slingshot: deploying stateful services in wireless hotspots. In 3rd international conference on Mobile systems, applications, and services (MobiSys), pages 79-92. ACM, 2005.

[30] D. Terry, M. Theimer, K. Petersen, A. Demers, M. Spreitzer, and C. Hauser. Managing update conflicts in bayou, a weakly connected replicated storage system. SIGOPS Oper. Syst. Rev., 29:172-182, December 1995. ISSN 0163-5980.

[31] E. Tilevich and Y. Smaragdakis. J-orchestra: Enhancing java programs with distribution capabilities. ACM Trans. Softw. Eng. Methodol., 19 (1):1:1-1:40, Aug. 2009. ISSN 1049-331X.

[32] N. Tillmann, M. Moskal, J. de Halleux, and M. Fahndrich. Touchdevelop: Programming cloud-connected mobile devices via touchscreen In 10th SIGPLAN Symposium on New Ideas, New Paradigms, and Reflections on Programming and Software (ONWARD), pages 49-60. ACM, 2011. ISBN 978-1-4503-0941-7.

[33] J. Wloka, M. Sridharan, and F. Tip. Refactoring for reentrancy. In 9th joint meeting of the European Software Engineering Conference and the ACM SIGSOFT Symposium on the Foundations of Software Engineering (ESEC/SIGSOFT FSE), pages 173-182, 2009.

[34] J. Wloka, M. Sridharan, and F. Tip. Refactoring for reentrancy. In 7th joint meeting of the European software engineering conference and the ACM SIGSOFT symposium on The foundations of software engineering (ESEC/FSE), pages 173-182. ACM, 2009.

[35] D. Wolber. App inventor and real-world motivation. In $42 N d A C M$ Technical Symposium on Computer Science Education (SIGCSE), pages 601-606. ACM, 2011.

[36] N. Yuill and Y. Rogers. Mechanisms for collaboration: A design and evaluation framework for multi-user interfaces. ACM Trans. Comput.Hum. Interact., 19(1):1:1-1:25, May 2012. ISSN 1073-0516. 